\title{
Selective branching and converting of topological modes
}

\author{
Toshikaze Kariyado ${ }^{1}{ }^{1}$ and Robert-Jan Slager $\oplus^{2}$ \\ ${ }^{1}$ International Center for Materials Nanoarchitectonics, National Institute for Materials Science, Tsukuba, Ibaraki 305-0044, Japan \\ ${ }^{2}$ TCM Group, Cavendish Laboratory, University of Cambridge, J. J. Thomson Avenue, Cambridge CB3 OHE, United Kingdom
}

(Received 25 March 2021; revised 8 July 2021; accepted 8 July 2021; published 6 August 2021)

\begin{abstract}
The existence of surface states is a salient feature of topological phases and many of the widely studied physical properties are directly tied to it. Although less explored, a variety of topological phases can be similarly distinguished by their response to localized flux defects, resulting in the binding of modes whose stability can be traced back to that of conventional edge states. The reduced dimensionality of these objects offers the possibility of arranging them in distinct geometries, such as arrays that branch or terminate in the bulk. We show that the prospect of hybridizing the modes in these kinds of channels results in new opportunities in a dynamical context. In particular, we find that certain branches of junctions of such extended defect arrays can be actively biased by manipulating initial conditions. Discussing these physical effects within a generally applicable framework that relates to a variety of established artificial topological materials, such as spring-mass setups and LC circuits, our results offer an avenue to explore and manipulate new transport effects that are rooted in the topological characterization of the underlying system.
\end{abstract}

DOI: 10.1103/PhysRevResearch.3.L032035

Introduction. With the discovery of time-reversal symmetry (TRS) invariant topological insulators (TIs) [1,2], the study of topological materials has become a prominent subject of interest, resulting in a plethora of phases and characterizations [3-21]. A notable signature of many topological phases is the bulk-boundary correspondence [22-33]. The anomalous edge states, circumventing the Nielsen-Ninomiya theorem [34], in many cases directly relate to sought-after physical signatures, ranging from axion behavior [35-40] to possible Majorana excitations [41], and accordingly also hold promise for future technological applications [42].

On another note, many TIs can also be characterized by their response to symmetry-preserving gauge fluxes that act as monodromy defects. Such fluxes bind midgap modes that in turn relate to the underlying topological invariant [16,43-52]. These gauge fluxes, which ideally has to have lattice constant scale localization, are generally hard to realize experimentally, although routes that use defects to mimic the effective response [49,53-57] are increasingly proving viable [58,59]. In this regard, the controllability of artificial materials [60] or cold atom systems [61,62] could provide a more immediate route to access this physics. Indeed, in a recent study we considered $\pi$-flux arrays in such metamaterial settings [63] and showed that the hybridization processes can be tuned to induce arbitrary flat semimetallic bands.

In this work, we take maximum advantage of both the highly tuneable nature of artificial materials and flexibility of

Published by the American Physical Society under the terms of the Creative Commons Attribution 4.0 International license. Further distribution of this work must maintain attribution to the author(s) and the published article's title, journal citation, and DOI. $\pi$-flux chains to uncover new dynamical effects. In contrast to normal interfaces, such chains in the bulk of topological materials offer different possibilities of terminating as well as branching them. We in particular consider the target geometries illustrated in Fig. 1. Namely, we discuss the dynamics in topological metamaterials across $\pi$-flux chain junctions [Fig. 1(a)] and also in geometries that connect $\pi$-flux chains to interface modes [Fig. 1(b)]. As a main result we find that, pending the initial conditions, the intensity across one branch can be substantially biased with regard to the other in the different setups, rather akin to a classical transistor in so much as the biasing of currents is concerned. Given the routinely implementable control of the parameters in the systems we discuss, we believe that our work can set a basis to experimentally explore these new bulk effects in a wider context.

Model and Method. In this Letter, we work on the modulated honeycomb lattice model, which is widely used in the context of topological metamaterials [64-72]. We first describe the quantum Hamiltonian $H$ and then explain how to relate it to metamaterials governed by a dynamical matrix $\hat{\Gamma}$. The Hamiltonian for the modulated honeycomb lattice model reads $H=\sum_{\langle i j\rangle} t_{i j} c_{i}^{\dagger} c_{j}$, in which $\langle i j\rangle$ indicates nearest neighbors and $c_{i}^{\dagger}$ refers to the creation operator at a site $i$. The hopping terms $t_{i j}$ feature a modulation set by $t_{0}=\bar{t}+\delta$ and $t_{1}=\bar{t}-2 \delta$. The former (latter) relates to the black (red) bonds in the schematic picture at the inset of Fig. 2(a). That is, with our modulation, a hexagonal six site cluster forms a unit, and the black and red bonds correspond to intra- and interunit hoppings, respectively. The Dirac cones of the pristine honeycomb model are then gapped out due to this modulation and the according mass term, which features a sign set by the sign of $\delta$. These gapped phases can in fact be characterized from a crystalline symmetry perspective using mirror winding numbers [73-75]. 
(a) junction

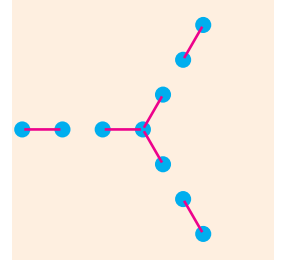

(b) interface

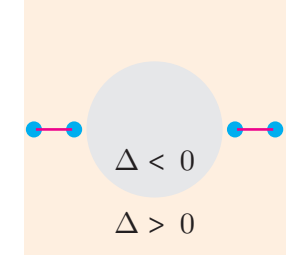

FIG. 1. Target geometries of $\pi$-flux modes (blue dots) that emerge at endpoints of hypothetical strings (magenta) across which hopping terms acquire a minus sign, see main text. (a) $\pi$-flux chain junction. (b) Interaction between $\pi$-flux chain and topological interface modes.

After fixing the hopping term geometry $t_{i j}, \pi$ fluxes are introduced by flipping the sign of the selected $t_{i j}$. This is achieved by placing hypothetical strings on the system, which change the sign of hoppings on the bonds crossing the string, resulting in $\pi$ fluxes at their endpoints. For the gapped phase with $\delta>0$, when $\pi$-flux threads through a hexagon of black $\left(t_{0}\right)$ bonds, it induces two in-gap modes per $\pi$ flux. These in-gap modes are protected by the sublattice (chiral) symmetry and the mirror symmetry whose reflection plane is perpendicular to the zigzag direction, related to the fact that the bulk topology is captured by mirror winding numbers. In other words, for an isolated $\pi$ flux, the two in-gap modes are indexed by the chiral and the reflection parity indices. Namely, one of the states has its wave-function weight exclusively on

(a)

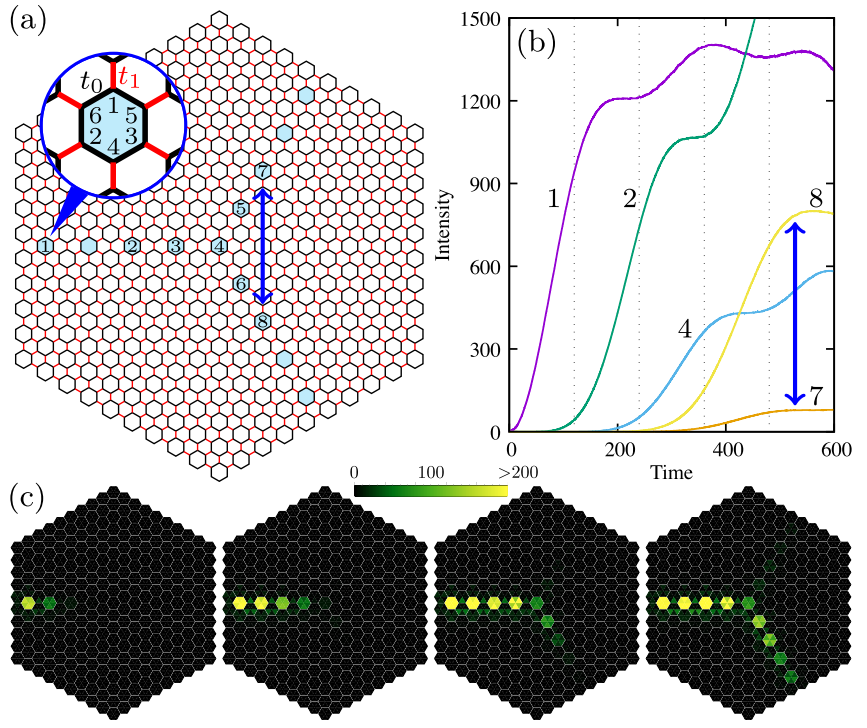

FIG. 2. (a) Schematic picture for a system with a $\pi$-flux chain junction. The cyan colored unit hexagons are threaded with $\pi$ fluxes. The system contains $13 \pi$ fluxes, and eight of them are numbered for convenience. The inset shows the unit-cell structure and the sublattice indices 1-6. (b) Time evolution of intensity $I_{i}^{\text {hex }}$ for $i$ th numbered hexagon. The intensities for the seventh and eighth hexagons show contrasting behavior. (c) Snapshots of the intensity map. The four shots correspond to the time slices indicated by the vertical dotted lines in panel (b). Bright color means high intensity. Majority of the energy from the source flows into the lower branch. the A sublattice [sublattices 1-3 in the inset of Fig. 2(a)] and is reflection-even, while the other has its weight exclusively on the B sublattice [sublattices 4-6 in the inset of Fig. 2(a)] and is reflection-odd (or vice versa) [63].

We remark that, in some studies of this type of modulated honeycomb lattice model, the $\delta<0$ phase is denoted topological. However, at the level of the effective Dirac theory, this concept is relative. It only indicates that the mass term changes its sign across the phase boundary between $\delta>0$ and $\delta<0$. Here, we consider $\pi$ fluxes in the $\delta>0$ phase, which resonates better with a reflection-symmetric placement of $\pi$ fluxes than in the $\delta<0$ phase in terms of generating the in-gap modes [63].

As a next step, we consider the dynamics governed by an equation of motion

$$
\frac{d^{2} x}{d t^{2}}=-\hat{\Gamma} x+f^{(c)} \cos \Omega t+f^{(s)} \sin \Omega t .
$$

That is, we assume that the system is characterized by a dynamical matrix $\hat{\Gamma}$ and follow the dynamics with an external monochromatic (single frequency) force. Here, $x$ is an $N$ dimensional vector for a system with $N$ degrees of freedom. The matrix elements of $\hat{\Gamma}$ are determined through $\Gamma_{i j}=\frac{\partial^{2} V}{\partial x_{i} \partial x_{j}}$ with

$$
V=\frac{1}{2} \sum_{i \in S} \sum_{j \in S, E} k_{i j}\left(x_{i}-x_{j}\right)^{2}+\frac{1}{2} \sum_{i \in S} \epsilon_{i} x_{i}^{2},
$$

where $i \in S$ represents the summation over sites. To apply a fixed boundary condition, which is important to preserve the sublattice symmetry of the honeycomb lattice at the boundary [76], the summation also runs over hypothetical external sites $E$ that have $x_{j}=0(j \in E)$. As a result, we then obtain

$$
\Gamma_{i j}=\left(\epsilon_{i}+\sum_{l \in S, E} k_{i l}\right) \delta_{i j}-k_{i j},
$$

where we match $k_{i j}$ to the Hamiltonian of the modulated honeycomb model with $\pi$ fluxes, i.e., we set $k_{i j}=t_{i j}$ so that the underlying background is indeed the modulated honeycomb model and adjust $\epsilon_{i}$ to make the diagonal term constant, giving only a trivial frequency shift.

From a physical point of view Eq. (1) describes any coupled harmonic-oscillator system. This covers, for instance, spring-mass models or LC circuits. Accordingly, $x$ may then represent the displacement of mass points, or the voltage at a certain point in a circuit, while $V$ plays the role of elastic energy in spring systems or charging energy of circuit elements. The platform can even be a continuum model like photonic crystal or elastic media, as far as it accepts an effective description by Eq. (1). Because of this wide applicability, we do not set any specific unit on $x$ and $t$, solving Eq. (1) as it is in the following.

The external force in Eq. (1) is introduced by singling out a $\pi$-flux threaded hexagon and applying $f_{0} \cos \Omega t\left(f_{0} \sin \Omega t\right)$ on the sublattice 1 (sublattice 4 ) in the unit hexagon. We note that this gives a quarter-period phase shift between the A-sublattice-localized $\pi$-flux mode and the B-sublatticelocalized $\pi$-flux mode, or, more specifically, the input on the $\mathrm{B}$ sublattices is a quarter-period phase advanced. Note that the 
quarter-period phase shift corresponds to the phase factor $i$ in quantum cases.

For the design of the $\pi$-flux placement, we work on the setups in Fig. 1. As we have noted, each $\pi$-flux threaded plaquette binds a pair of modes and, upon making a $\pi$-flux chain, there arises a one-dimensional (1D) propagating channel. We analyze the dynamics, how energy passed from the external forces propagates in the given system, by mainly focusing on the quantity

$$
I_{i}=\Omega^{2} x_{i}^{2}+\left(\frac{d x_{i}}{d t}\right)^{2}
$$

which roughly corresponds to an intensity on each site and hence allows for tracking of the effective dynamics [77]. Practically, we find that this combination of the first and the second terms eliminates the rapid oscillation well. For convenience, we accordingly also make use of $I_{a}^{\text {hex }}$, which is defined as the sum of $I_{i}$ within a unit hexagon specified by an index $a$.

In all cases below, we assume that the system is at rest at $t=0$. Also, we always use hexagon 1 to inject energy with the quarter-period phase shift between the A and B sublattices, as noted above. For the hopping parameters, we set $\bar{t}=1$ and $|\delta|=0.2$. Then, we fix the frequency $\Omega$ of the external force as $\Omega=\sqrt{3}+0.01$, where $\sqrt{3}$ is the middle of the bulk gap corresponding to $\{\bar{t},|\delta|\}=\{1,0.2\}$, and 0.01 is added to prevent possible nonessential effects by the mixed right movers and left movers. With this setup, the period of the rapid oscillation $2 \pi / \Omega$ is approximately 3.6. Equation (1) is solved by normal-mode decomposition (see Supplemental Materials [78]), and cross-checked by the Runge-Kutta method. The solution of Eq. (1) is proportional to $f_{0}$, and the following results are calculated with $f_{0}=1$.

Results. Turning first to the junction, we find the numerical results displayed in Fig. 2. The intensity is transmitted from the source (hexagon 1) to the right along the $\pi$-flux chain [Figs. 2(b) and 2(c)]. Interestingly, after passing the junction, the intensity shows a biased behavior; the lower branch has significantly larger intensity than the upper branch. We have checked that the bias is inverted if the force on the B sublattice in hexagon 1 is delayed by a quarter period phase rather than advanced, and that there is no bias without the phase shift in the input (see Supplemental Materials [78]). That is, the fate of the $\pi$-flux mode is controlled by the local manipulation of the input at the source. This actively biased behavior can be potentially used as a building block for new devices. Some parameter or perturbation dependence regarding the robustness of the effect is detailed in the Supplemental Materials [78].

To understand this phenomenon qualitatively, let us consider how the intensity is transmitted from hexagon 3 to hexagon 5 or 6 over the junction. Owing to the chiral symmetry of the model, the only relevant matrix elements are those coupling the A and B sublattices. Therefore, starting from hexagon 3, the intensity can be passed to the A sublattice sites of hexagon 6 in two ways. Either via the process $h_{\mathrm{A}}^{3} \rightarrow h_{\mathrm{B}}^{4} \rightarrow h_{\mathrm{A}}^{6}$ or $h_{\mathrm{B}}^{3} \rightarrow h_{\mathrm{A}}^{6}$, where $h_{\alpha}^{j}$ denotes the $\alpha=\mathrm{A}, \mathrm{B}$ sublattice of hexagon $j$. Together with the phase acquired along the real-space paths, the inherent quarter-period phase shift between A and B sublattices at the origin leads to the constructive interference at hexagon 6. Furthermore, if we try

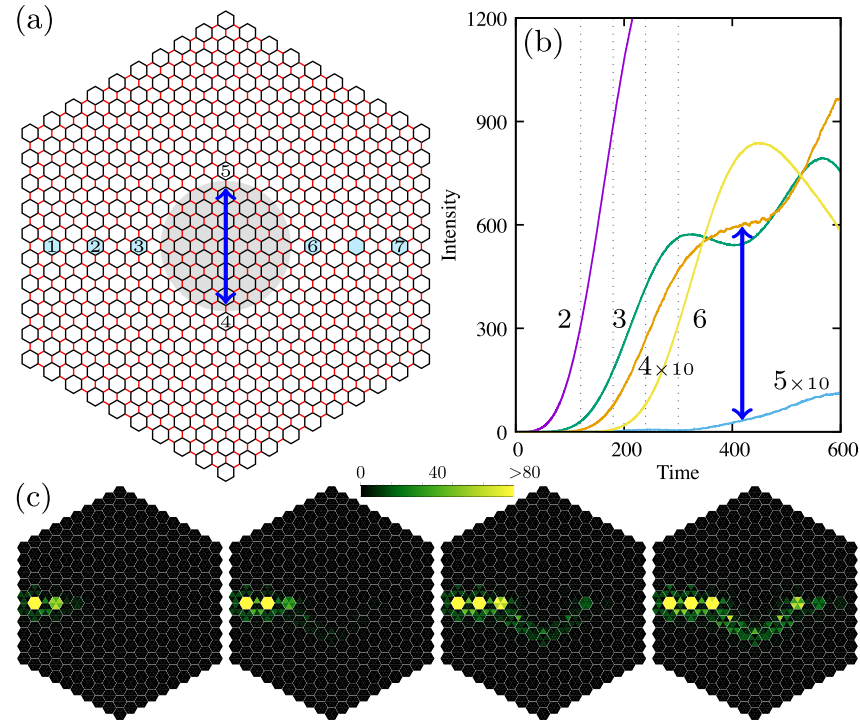

FIG. 3. (a) System with $\pi$-flux chains and a topological interface. In the circular shaded region at the center, we set $\delta=-0.2$, while in the other region, we set $\delta=0.2$. (b) Time evolution of intensity $I_{i}^{\text {hex }}$ for $i$ th numbered hexagon. For better comparison, $I_{4}^{\text {hex }}$ and $I_{5}^{\text {hex }}$ are scaled by a factor of ten. The intensities for the fourth and fifth hexagons show contrasting behavior. (c) Snapshots of the intensity map. The four shots correspond to the time slices indicated by the vertical dotted lines in panel (b). The majority of the energy from the source flows along the lower half of the interface.

to relate hexagon 5 and 6 , the roles of the A and B sublattices are interchanged due to the lattice geometry, and the advance (delay) is reinterpreted as delay (advance). This consequently results in destructive interference at hexagon 5, concluding the essential explanation underpinning the bias between the branches. The same course of thinking also elucidates why the bias is inverted or suppressed upon changing the phase shift at the source. This relation furthermore brings to light that the biasing ability is determined by the ratio between the coupling of $h_{\mathrm{A}, \mathrm{B}}^{3} \rightarrow h_{\mathrm{B}, \mathrm{A}}^{4} \rightarrow h_{\mathrm{A}, \mathrm{B}}^{5,6}$ and $h_{\mathrm{A}, \mathrm{B}}^{3} \rightarrow h_{\mathrm{B}, \mathrm{A}}^{5,6}$, which will be affected by the bulk gap.

Crucially, this analysis can be enforced by analyzing the descriptive model, named the projected model as detailed in the Supplemental Materials [78]. There we construct an effective tight-binding model for $\pi$-flux modes by projecting the system onto the subspace spanned by the in-gap states. This procedure conveys the effective hoppings between the $\pi$ fluxes, which reproduces satisfactorily the dynamics of the original model. Eliminating some of the hopping processes in the projected model by hand, it is then readily confirmed that, when the hoppings between the hexagon 3, 5, and 6 are turned off, the biasing behavior is also turned off. This is in line with the above consideration.

Next, we consider situations involving the $\pi$-flux chain and interface states, as in the setup of Fig. 3(a). The parameter $\delta$ is positive outside the gray shaded region, as before, while $\delta$ is negative inside the gray shaded region, and we expect to have interface modes at this interface. The $\pi$ fluxes are again threading the cyan colored hexagons. The resulting time evolution of the intensity is summarized in Figs. 3(b) and 3(c). 
One thing we notice is that the intensity propagates faster along the interface than along the $\pi$-flux chain, indicated by the timing of the intensity growth at hexagons 3 and 4 . The difference in the group velocity along the interface and along the $\pi$-flux chain will generally depend on the bulk-gap size. The other, more striking thing that we notice is that there is a significant difference between the intensity at hexagon 4 and hexagon 5. In Fig. 3(c), we can see that the intensity propagates along the lower half of the circular interface. As in the case of biasing the $\pi$-flux chain junction, the way that intensity branches is linked to the phase shift at the source. If delay and advance is exchanged, the intensity goes around the upper half of the interface, or if there is no phase shift, the intensity splits equally to the upper and the lower halves of the interface (see Supplemental Materials [78]). We conclude that, as in the junction, we can bias branching channels, now involving edge states degrees of freedom, by manipulating the initial condition at the source offering new switch effects in the bulk of the topological material. To understand this branching, it is essential to know the overlap between the wave functions for the $\pi$-flux modes and the interface modes. For the $\pi$-flux modes, the wave functions can be inferred from the results in the isolated $\pi$-flux case [63]. For the interface modes, a rough description of the wave functions is obtained by approximating the bulk states by a $k \cdot p$-type continuous model (see Supplemental Materials [78]). It turns out that the phase delay or advance in the $\pi$-flux modes effectively selects the right or left movers in the interface states to couple.

Conclusion and discussion. In conclusion, we have shown that the versatility of $\pi$ fluxes in topological meta-materials leads to new dynamical effects. In particular, we find that branched channels can be designed to show biased behavior with the initial condition as a switch. Interestingly, this effect is rooted in the topology of the underlying system.

These mechanisms maximally profit from the engineerability, positively impacting the implementability in three ways: First, the modulated honeycomb system can be readily realized in disk-spring systems or LC circuits. Second, these systems offer realistic handling of the initial conditions being the control parameter to establish and manipulate the branching effects. Third, they offer a rather feasible route to create $\pi$-flux threaded plaquettes. Indeed, Ref. [79], for instance, points out how one could control the hopping sign essential for implementing such fluxes by using reversed springs. Similarly, specific kinds of wiring as proposed in Ref. [80] induce a route to implement them in LC circuits. Finally, we point out that yet another route is to control the potential of neighbors, meaning that nearest hopping terms can be replaced by an intermediate site [63]. Adjusting the relative strength of the potential can then induce a sign flip with respect to the initial hopping process.
In experimental implementations, dissipation is unavoidable. A major effect of finite dissipation is to shorten the propagation distance along the $\pi$-flux chains. We can redesign the bulk parameters to keep the propagation distance sufficiently long, according to the strength of dissipation (see Supplemental Materials [78]). Another interesting topic is to explore non-Hermitian physics with $\pi$ fluxes, which is left as a future problem.

Since the essence of the dynamics of the in-gap mode is the hopping between the localized modes, one may wonder what is the benefit of embedding $\pi$ fluxes in a topological system over arranging trivial (local) resonators or oscillators. First of all, if a phenomenon has topology behind, we expect that it is universal in a variety of implementations. Also, the $\pi$-flux-based setup has another benefit in relation to interface modes. Namely, the existence of the $\pi$-flux modes at the same gap and at the same frequency as the interface modes is guaranteed since they share the same origin [63]. It is worth noting that the interface modes in the current model have been experimentally observed, and their robustness against backscattering originated from topology has also been confirmed [69]. Then, the next step would be to build some working devices, and there the cross coupling between the $\pi$-flux modes and the interface modes will open interesting possibilities, and it is advantageous that there is no need for fine tuning to enjoy that cross coupling. Second, with this construction, we automatically have two bound states per single $\pi$ flux [63], and we take full advantage of the quarter-period phase shift between the two local modes to realize unbalanced branching. Also, this double degeneracy naturally induces Dirac-type band dispersion upon making a 1D channel with $\pi$-flux arrays. Last but not least, the design of $\pi$-flux chains is more flexible than interface modes; e.g., $\pi$-flux chains can form a Y-shaped junction, can bridge two separated interfaces, and can be terminated, all of which are not possible with interfaces only. Additionally, to change the geometry of 1D channels of the $\pi$-flux chains, we only have to relocate the $\pi$-phase string, while for interfaces, we have to change the all relevant parameters in an area surrounded by the original and the new interfaces. Finally, another setup in which the chain is dynamically extended, thereby leading to a stop-and-go effect, is presented in the Supplemental Materials [78], showing the versatility of our setup.

Acknowledgments. R.-J.S. acknowledges funding via the Marie Skłodowska-Curie programm under EC Grant No. 842901 and the Winton programme as well as Trinity College at the University of Cambridge. This work was furthermore supported by JSPS KAKENHI Grant No. JP17K14358 (T.K.). The part of computation in this work has been done using the facilities of the Supercomputer Center, the Institute for Solid State Physics, the University of Tokyo.
[1] M. Z. Hasan and C. L. Kane, Colloquium: Topological insulators, Rev. Mod. Phys. 82, 3045 (2010).

[2] X.-L. Qi and S.-C. Zhang, Topological insulators and superconductors, Rev. Mod. Phys. 83, 1057 (2011).

[3] A. Kitaev, Periodic table for topological insulators and superconductors, in Advances in Theoretical Physics: Landau Memo- rial Conference, edited by V. Lebedev and M. Feigel'man, AIP Conf. Proc. 1134 (AIP, Melville, NY, 2009), p. 22.

[4] S. Ryu, A. P. Schnyder, A. Furusaki, and A. W. W. Ludwig, Topological insulators and superconductors: Tenfold way and dimensional hierarchy, New J. Phys. 12, 065010 (2010). 
[5] L. Fu, Topological Crystalline Insulators, Phys. Rev. Lett. 106, 106802 (2011).

[6] R.-J. Slager, A. Mesaros, V. Juričić, and J. Zaanen, The space group classification of topological band-insulators, Nat. Phys. 9, 98 (2012).

[7] K. Shiozaki and M. Sato, Topology of crystalline insulators and superconductors, Phys. Rev. B 90, 165114 (2014).

[8] T. L. Hughes, E. Prodan, and B. A. Bernevig, Inversionsymmetric topological insulators, Phys. Rev. B 83, 245132 (2011).

[9] A. M. Turner, Y. Zhang, R. S. K. Mong, and A. Vishwanath, Quantized response and topology of magnetic insulators with inversion symmetry, Phys. Rev. B 85, 165120 (2012).

[10] J. Kruthoff, J. de Boer, J. van Wezel, C. L. Kane, and R.-J. Slager, Topological Classification of Crystalline Insulators Through Band Structure Combinatorics, Phys. Rev. X 7, 041069 (2017).

[11] H. C. Po, A. Vishwanath, and H. Watanabe, Symmetry-based indicators of band topology in the 230 space groups, Nat. Commun. 8, 50 (2017).

[12] A. Bouhon and A. M. Black-Schaffer, Global band topology of simple and double Dirac-point semimetals, Phys. Rev. B 95, 241101(R) (2017).

[13] B. Bradlyn, L. Elcoro, J. Cano, M. G. Vergniory, Z. Wang, C. Felser, M. I. Aroyo, and B. A. Bernevig, Topological quantum chemistry, Nature (London) 547, 298 (2017).

[14] F. N. Ünal, A. Bouhon, and R.-J. Slager, Topological Euler Class as a Dynamical Observable in Optical Lattices, Phys. Rev. Lett. 125, 053601 (2020).

[15] A. Alexandradinata, J. Höller, C. Wang, H. Cheng, and L. Lu, Crystallographic splitting theorem for band representations and fragile topological photonic crystals, Phys. Rev. B 102, 115117 (2020).

[16] R.-J. Slager, The translational side of topological band insulators, J. Phys. Chem. Solids 128, 24 (2019).

[17] X. Wan, A. M. Turner, A. Vishwanath, and S. Y. Savrasov, Topological semimetal and Fermi-arc surface states in the electronic structure of pyrochlore iridates, Phys. Rev. B 83, 205101 (2011).

[18] A. Bouhon, A. M. Black-Schaffer, and R.-J. Slager, Wilson loop approach to fragile topology of split elementary band representations and topological crystalline insulators with time-reversal symmetry, Phys. Rev. B 100, 195135 (2019).

[19] A. Bouhon, G. F. Lange, and R.-J. Slager, Topological correspondence between magnetic space group representations and subdimensions, Phys. Rev. B 103, 245127 (2021).

[20] T. Bzdušek, Q. Wu, A. Rüegg, M. Sigrist, and A. A. Soluyanov, Nodal-chain metals, Nature (London) 538, 75 (2016).

[21] B.-J. Yang and N. Nagaosa, Classification of stable threedimensional Dirac semimetals with nontrivial topology, Nat. Commun. 5, 4898 (2014).

[22] E. Prodan and H. Schulz-Baldes, Bulk and Boundary Invariants for Complex Topological Insulators (Springer International Publishing, Cham, Switzerland, 2016).

[23] Y. Hatsugai, Chern Number and Edge States in the Integer Quantum Hall Effect, Phys. Rev. Lett. 71, 3697 (1993).

[24] E. Khalaf, W. A. Benalcazar, T. L. Hughes, and R. Queiroz, Boundary-obstructed topological phases, Phys. Rev. Res. 3, 013239 (2021).
[25] R.-J. Slager, L. Rademaker, J. Zaanen, and L. Balents, Impurity-bound states and Green's function zeros as local signatures of topology, Phys. Rev. B 92, 085126 (2015).

[26] J.-W. Rhim, J. H. Bardarson, and R.-J. Slager, Unified bulkboundary correspondence for band insulators, Phys. Rev. B 97, 115143 (2018).

[27] D. S. Borgnia, A. J. Kruchkov, and R.-J. Slager, Non-Hermitian Boundary Modes and Topology, Phys. Rev. Lett. 124, 056802 (2020).

[28] Z. Song, Z. Fang, and C. Fang, $(d-2)$-Dimensional Edge States of Rotation Symmetry Protected Topological States, Phys. Rev. Lett. 119, 246402 (2017).

[29] W. A. Benalcazar, B. A. Bernevig, and T. L. Hughes, Quantized electric multipole insulators, Science 357, 61 (2017).

[30] D. Călugăru, V. Juričić, and B. Roy, Higher-order topological phases: A general principle of construction, Phys. Rev. B 99, 041301(R) (2019).

[31] L. Trifunovic and P. W. Brouwer, Higher-order topological band structures, Phys. Status Solidi B 258, 2000090 (2021).

[32] G. F. Lange, A. Bouhon, and R.-J. Slager, Subdimensional topologies, indicators, and higher order boundary effects, Phys. Rev. B 103, 195145 (2021).

[33] F. Schindler, Z. Wang, M. G. Vergniory, A. M. Cook, A. Murani, S. Sengupta, A. Y. Kasumov, R. Deblock, S. Jeon, I. Drozdov, H. Bouchiat, S. Guéron, A. Yazdani, B. A. Bernevig, and T. Neupert, Higher-order topology in bismuth, Nat. Phys. 14, 918 (2018).

[34] H. Nielsen and M. Ninomiya, A no-go theorem for regularizing chiral fermions, Phys. Lett. B 105, 219 (1981).

[35] X.-L. Qi, T. L. Hughes, and S.-C. Zhang, Topological field theory of time-reversal invariant insulators, Phys. Rev. B 78, 195424 (2008).

[36] N. H. Jo, L.-L. Wang, R.-J. Slager, J. Yan, Y. Wu, K. Lee, B. Schrunk, A. Vishwanath, and A. Kaminski, Intrinsic axion insulating behavior in antiferromagnetic $\mathrm{MnBi}_{6} \mathrm{Te}_{10}$, Phys. Rev. B 102, 045130 (2020).

[37] B. J. Wieder and B. A. Bernevig, The axion insulator as a pump of fragile topology, arXiv:1810.02373.

[38] C. Liu, Y. Wang, H. Li, Y. Wu, Y. Li, J. Li, K. He, Y. Xu, J. Zhang, and Y. Wang, Robust axion insulator and Chern insulator phases in a two-dimensional antiferromagnetic topological insulator, Nat. Mater. 19, 522 (2020).

[39] R. S. K. Mong, A. M. Essin, and J. E. Moore, Antiferromagnetic topological insulators, Phys. Rev. B 81, 245209 (2010).

[40] M. M. Otrokov, I. I. Klimovskikh, H. Bentmann, D. Estyunin, A. Zeugner, Z. S. Aliev, S. Gaß, A. U. B. Wolter, A. V. Koroleva, A. M. Shikin, M. Blanco-Rey, M. Hoffmann, I. P. Rusinov, A. Y. Vyazovskaya, S. V. Eremeev, Y. M. Koroteev, V. M. Kuznetsov, F. Freyse, J. Sánchez-Barriga, I. R. Amiraslanov et al., Prediction and observation of an antiferromagnetic topological insulator, Nature (London) 576, 416 (2019).

[41] L. Fu and C. L. Kane, Superconducting Proximity Effect and Majorana Fermions at the Surface of a Topological Insulator, Phys. Rev. Lett. 100, 096407 (2008).

[42] J. E. Moore, The birth of topological insulators, Nature (London) 464, 194 (2010).

[43] R. Jackiw and P. Rossi, Zero modes of the vortex-fermion system, Nucl. Phys. B 190, 681 (1981). 
[44] E. J. Weinberg, Index calculations for the fermion-vortex system, Phys. Rev. D 24, 2669 (1981).

[45] C.-Y. Hou, C. Chamon, and C. Mudry, Electron Fractionalization in Two-Dimensional Graphenelike Structures, Phys. Rev. Lett. 98, 186809 (2007).

[46] B. Seradjeh, C. Weeks, and M. Franz, Fractionalization in a square-lattice model with time-reversal symmetry, Phys. Rev. B 77, 033104 (2008).

[47] Y. Ran, A. Vishwanath, and D.-H. Lee, Spin-Charge Separated Solitons in a Topological Band Insulator, Phys. Rev. Lett. 101, 086801 (2008).

[48] X.-L. Qi and S.-C. Zhang, Spin-Charge Separation in the Quantum Spin Hall State, Phys. Rev. Lett. 101, 086802 (2008).

[49] V. Juričić, A. Mesaros, R.-J. Slager, and J. Zaanen, Universal Probes of Two-Dimensional Topological Insulators: Dislocation and $\pi$ Flux, Phys. Rev. Lett. 108, 106403 (2012).

[50] A. Mesaros, R.-J. Slager, J. Zaanen, and V. Juričić, Zero-energy states bound to a magnetic $\pi$-flux vortex in a two-dimensional topological insulator, Nucl. Phys. B 867, 977 (2013).

[51] X.-G. Wen, Colloquium: Zoo of quantum-topological phases of matter, Rev. Mod. Phys. 89, 041004 (2017).

[52] Y.-J. Wu, J. He, and S.-P. Kou, Topological mid-gap states of Chern insulator with flux-superlattice, Europhys. Lett. 105, 47002 (2014).

[53] Y. Ran, Y. Zhang, and A. Vishwanath, One-dimensional topologically protected modes in topological insulators with lattice dislocations, Nat. Phys. 5, 298 (2009).

[54] K.-I. Imura, Y. Takane, and A. Tanaka, Weak topological insulator with protected gapless helical states, Phys. Rev. B 84, 035443 (2011).

[55] R. Queiroz, I. C. Fulga, N. Avraham, H. Beidenkopf, and J. Cano, Partial Lattice Defects in Higher-Order Topological Insulators, Phys. Rev. Lett. 123, 266802 (2019).

[56] R.-J. Slager, V. Juričić, V. Lahtinen, and J. Zaanen, Selforganized pseudo-graphene on grain boundaries in topological band insulators, Phys. Rev. B 93, 245406 (2016).

[57] R.-J. Slager, A. Mesaros, V. Juričić, and J. Zaanen, Interplay between electronic topology and crystal symmetry: Dislocation-line modes in topological band insulators, Phys. Rev. B 90, 241403(R) (2014).

[58] H. Hamasaki, Y. Tokumoto, and K. Edagawa, Dislocation conduction in Bi-Sb topological insulators, Appl. Phys. Lett. 110, 092105 (2017).

[59] A. K. Nayak, J. Reiner, R. Queiroz, H. Fu, C. Shekhar, B. Yan, C. Felser, N. Avraham, and H. Beidenkopf, Resolving the topological classification of bismuth with topological defects, Sci. Adv. 5, eaax6996 (2019).

[60] S. D. Huber, Topological mechanics, Nat. Phys. 12, 621 (2016).

[61] J. Dalibard, F. Gerbier, G. Juzeliūnas, and P. Öhberg, Colloquium: Artificial gauge potentials for neutral atoms, Rev. Mod. Phys. 83, 1523 (2011).

[62] B. Wang, F. N. Ünal, and A. Eckardt, Floquet Engineering of Optical Solenoids and Quantized Charge Pumping Along Tailored Paths in Two-Dimensional Chern Insulators, Phys. Rev. Lett. 120, 243602 (2018).

[63] T. Kariyado and R.-J. Slager, $\pi$-fluxes, semimetals, and flat bands in artificial materials, Phys. Rev. Res. 1, 032027(R) (2019).

[64] S. Yves, R. Fleury, T. Berthelot, M. Fink, F. Lemoult, and G. Lerosey, Crystalline metamaterials for topological properties at subwavelength scales, Nat. Commun. 8, 16023 (2017).

[65] S. Barik, A. Karasahin, C. Flower, T. Cai, H. Miyake, W. DeGottardi, M. Hafezi, and E. Waks, A topological quantum optics interface, Science 359, 666 (2018).

[66] S.-Y. Yu, C. He, Z. Wang, F.-K. Liu, X.-C. Sun, Z. Li, H.-Z Lu, M.-H. Lu, X.-P. Liu, and Y.-F. Chen, Elastic pseudospin transport for integratable topological phononic circuits, Nat. Commun. 9, 3072 (2018).

[67] Y. Li, Y. Sun, W. Zhu, Z. Guo, J. Jiang, T. Kariyado, H. Chen, and $\mathrm{X}$. Hu, Topological LC-circuits based on microstrips and observation of electromagnetic modes with orbital angular momentum, Nat. Commun. 9, 4598 (2018).

[68] J. Noh, W. A. Benalcazar, S. Huang, M. J. Collins, K. P. Chen, T. L. Hughes, and M. C. Rechtsman, Topological protection of photonic mid-gap defect modes, Nat. Photonics 12, 408 (2018).

[69] Y. Yang, Y. F. Xu, T. Xu, H.-X. Wang, J.-H. Jiang, X. Hu, and Z. H. Hang, Visualization of a Unidirectional Electromagnetic Waveguide Using Topological Photonic Crystals Made of Dielectric Materials, Phys. Rev. Lett. 120, 217401 (2018).

[70] D. V. Zhirihin, M. A. Gorlach, X. Ni, D. A. Smirnova, D. Korobkin, A. P. Slobozhanyuk, P. A. Belov, A. Alù, and A. B. Khanikaev, Experimental observation of spin-locked propagation of topological edge states in an open non-Hermitian metasurface, J. Phys.: Conf. Ser. 1092, 012176 (2018).

[71] R. Chaunsali, C.-W. Chen, and J. Yang, Experimental demonstration of topological waveguiding in elastic plates with local resonators, New J. Phys. 20, 113036 (2018).

[72] S. E. Freeney, J. J. van den Broeke, A. J. J. Harsveld van der Veen, I. Swart, and C. Morais Smith, Edge-Dependent Topology in Kekulé Lattices, Phys. Rev. Lett. 124, 236404 (2020).

[73] L.-H. Wu and X. Hu, Scheme for Achieving a Topological Photonic Crystal by Using Dielectric Material, Phys. Rev. Lett. 114, 223901 (2015).

[74] L.-H. Wu and X. Hu, Topological properties of electrons in honeycomb lattice with detuned hopping energy, Sci. Rep. 6, 24347 (2016).

[75] T. Kariyado and X. Hu, Topological states characterized by mirror winding numbers in graphene with bond modulation, Sci. Rep. 7, 16515 (2017).

[76] T. Kariyado and Y. Hatsugai, Manipulation of Dirac cones in mechanical graphene, Sci. Rep. 5, 18107 (2015).

[77] This intensity is almost local energy. However, in the system described by Eq. (2), the elastic or charging energy is stored on the bonds rather than the sites. We introduce Eq. (2) for conceptual simplicity, since it only requires information from a single site.

[78] See Supplemental Materials at http://link.aps.org/supplemental/ 10.1103/PhysRevResearch.3.L032035 for the numerical details, the derivation of an effective model, the phase shift effects, the introduction of the quench and termination setup, a detailed treatment of the robustness of the effects, and an analytical treatment of the coupling of edge and branch modes.

[79] R. Kumar, M. Schaeffer, and M. Ruzzene, Helical edge states and topological phase transitions in phononic systems using bilayered lattices, J. Appl. Phys. 119, 084305 (2016).

[80] Y. Lu, N. Jia, L. Su, C. Owens, G. Juzeliūnas, D. I. Schuster, and J. Simon, Probing the Berry curvature and Fermi arcs of a Weyl circuit, Phys. Rev. B 99, 020302(R) (2019). 